The Japan Society of Human Genetics Junior Award Lecture

\title{
NONKETOTIC HYPERGLYCINEMIA: BIOCHEMICAL, MOLECULAR, AND NEUROLOGICAL ASPECTS
}

\author{
Shigeo KURE,* Keiya TADA, and Kuniaki NARISAwA \\ Department of Pediatrics and Biochemical Genetics, Tohoku University \\ School of Medicine, 1-1 Seiryo-machi, Aoba-ku, Sendai 980-77, Japan
}

\begin{abstract}
Summary Nonketotic hyperglycinemia (NKH) is a metabolic disorder with autosomal recessive inheritance, causing severe, frequently lethal, neurological symptoms in the neonatal period. The metabolic lesion of NKH is in the glycine cleavage system (GCS), a complex enzyme system with four enzyme components; P-, T-, H-, and L-protein. The enzymatic analysis revealed that $86 \%$ of the patients with NKH are deficient of P-protein activity. The cDNA clones encoding all four components were isolated and their primary structures were determined. Several mutations have been identified in P- and T-protein genes: One missense mutation, S564I, in P-protein gene accounts for $70 \%$ of the mutant alleles in Finland where the incidence of NKH is unusually high. The immunochemical and in situ hybridization analyses revealed that the strong GCS expression was observed in rat hippocampus, olfactory bulbus, and cerebellum. The distribution resembled that of $N$-methylD-aspartic acid (NMDA) receptor which has binding site for glycine. It is, therefore, suggested that the neurological disturbance in NKH may be caused by excitoneurotoxicity through the NMDA receptor allosterically activated by high concentration of glycine. Based on the hypothesis the NMDA antagonists such as ketamine and dextromethorphan were administered to the patients. We treated three neonatal case with dextromethorphan and it ameliorated their findings on electroencephalogram and behavior in two out of three patients. Thus the GCS is suggested to play a role in regulation of glycine level around the NMDA receptor.
\end{abstract}

\section{Introduction}

Nonketotic hyperglycinemia (NKH) is an autosomal recessive disorder of

* To whom correspondence should be addressed.

Abbreviations: NKH, nonketotic hyperglycinemia; GCS, glycine cleavage system; PCR, polymerase chain reaction; EEG, electroencephalogram: NMDA, $N$-methyl-D-aspartic acid. 
glycine metabolism, characterized by abnormally high glycine concentrations of plasma and cerebrospinal fluid. Almost patients with NKH have their symptoms in neonatal period. Patients with neonatal onset NKH have myoclonic seizures, hypotonia, respiratory distress and lethargy within a few days after birth (Tada and Hayasaka, 1987; Hamosh et al., 1995). The mortality rate is high and survivors are severely retarded. Many patients must die before benefit of the diagnosis because of rapid deterioration of its clinical courses. The clinical presentations of this diseases are considered to be heterogeneous. Seizures and respiratory distress are not common in the late onset cases. NKH is a rare disease in many countries. In northern Finland, however, the prevalence of NKH is reported to be $1: 12,000$ birth, which is much more higher than the other countries (v. Wendt et al., 1979).

The primary lesion in NKH resides in the glycine cleavage system (GCS) with mitochondrial multienzyme components (EC 2.1.2.10) (Tada et al., 1969). The studies of $\mathrm{NKH}$ has elucidated the physiological significance of the GCS: It operates as a main degradation pathway of glycine in the vertebrate. Since almost patients with NKH has been identified to be deficiency of P-protein, a components of the GCS, we will focus on the biochemical and molecular aspects of P-protein deficiency. We will also describe the recent studies on neuropathogenesis of $\mathrm{NKH}$.

\section{Glycine cleavage system}

Enzymology. The mechanism of the reaction of the GCS is extensively studied by the research group of Motokawa and Kikuchi (Motokawa and Kikuchi, 1972; Kikuchi, 1973; Kikuchi et al., 1980). The GCS catalyzes the conversion of glycine and tetrahydrofolate to $\mathrm{CO}_{2}, \mathrm{NH}_{3}$, and methylene-tetrahydrofolate. The GCS is a mitochondrial multienzyme system with four individual components (Table 1); P-protein, glycine dehydrogenase (decarboxylating) (EC 1.4.4.2); Hprotein, hydrogen carrier protein; T-protein, aminomethyltransferase (EC 2.1.2. 10); and L-protein, dihydrolipoamide dehydrogenase (EC 1.8.1.4). In degradation of glycine by the GCS, P- and H-protein contribute to the initial step of decarboxylation. Glycine forms a Shiff base with the pyridoxal phosphate in P-protein, then the disulfide form of $\mathrm{H}$-protein combines with this complex, liberating $\mathrm{CO}_{2}$ from carboxyl moiety of glycine. This step is coupled with reduction of the disulfide group of $\mathrm{H}$-protein. The $\alpha$-carbon of glycine binds immediately to the $\mathrm{H}$-protein to form an S-C bond without loss of hydrogen atoms which attached to the $\alpha$-carbon. The $\mathrm{H}$-protein with the intermediate is then decomposed to yield methylene tetrahydrofolate and ammonia and the H-protein becomes a dithiol form. The dithiol form of $\mathrm{H}$-protein is reoxidized to the disulfide form by $\mathrm{L}$ protein and $\mathrm{NAD}^{+}$. L-protein is also used as $\mathrm{E} 3$ component of other multienzyme system such as pyruvate dehydrogenase complex. The symptoms of L-protein deficiency resemble those of pyruvate dehydrogenase deficiency and there is a report that L-protein dificiency caused Leigh disease (Craigen, 1996). In this paper we will describe only $\mathrm{P}$-, $\mathrm{H}$-, and $\mathrm{T}$-proteins which are unique components for the 
Table 1. Components of glycine cleavage system.

\begin{tabular}{lllll}
\hline Abbreviation & \multicolumn{1}{c}{ Enzyme name } & Co-factor & $\begin{array}{l}\text { Subunit } \\
\text { (amino acid) }\end{array}$ & $\begin{array}{c}\text { Chromosomal } \\
\text { location }\end{array}$ \\
\hline P-protein & $\begin{array}{l}\text { glycine } \\
\text { dehydrogenase } \\
\text { (decarboxylating) } \\
\text { (hydrogen carrier } \\
\text { Hotein) }\end{array}$ & $\begin{array}{l}\text { pyridoxal } \\
\text { phosphate }\end{array}$ & $\begin{array}{l}\text { Homodimer } \\
(1,024 \text { a.a. })\end{array}$ & $9 \mathrm{p} 24$ \\
T-protein & Lipoic acid & $\begin{array}{l}\text { Monomer } \\
(173 \text { a.a. })\end{array}$ & - \\
L-protein & $\begin{array}{l}\text { dihydrolipoamide } \\
\text { dehydrogenase }\end{array}$ & FAD & $\begin{array}{l}\text { (403 a.a. }) \\
\text { Homodimer } \\
(509 \text { a.a. })\end{array}$ & - \\
\hline
\end{tabular}

GCS.

Structure of the GCS. The cDNAs encoding all four components of GCS were isolated and their primary structures were determined (Table 1). Human P-protein cDNA clones were isolated from $\lambda$ gt 11 expression libraries with specific antibodies (Kume et al, 1991; Kure et al, 1991b). Its genomic counterpart mapped to chromosome 9 and consisted of 25 exons. The human P-protein subunit precursor was deduced to contain 1,020 amino acids and have a mitochondrial leader peptide of 39 amino acids. The cofactor of P-protein pyridoxal phosphate binds to ${ }^{715}$ Lys of human P-protein precursor (Fujiwara et al., 1987). The authenticity of this clones was verified by an expression study of the cDNA clone. When the clone was expressed in COS7 cells, the specific activity of P-protein in the COS7 cell extract was markedly elevated (Kure et al., 1991b). Besides of human P-protein, cDNA clones of chicken (Kume et al., 1991) and Escherichia coli (Okamura-Ikeda et al., 1993) were isolated. Amino acid sequence homology between human and chicken is $83 \%$ and that between human and E. coli is $53 \%$. The homology of the mitochondrial leader peptides of human and chicken is very low. Molecular cloning of T-protein cDNAs revealed that $\mathrm{T}$-protein precursor consists of 397 amino acids in bovine (Okamura-Ikeda et al., 1991) and 403 amino acids in human (Hayasaka et al., 1993). Its genomic counterpart has nine exons and mapped to chromosome 3 (Nanao et al., 1994). The primary structure of a mature form of chicken $\mathrm{H}$-protein was determined by the peptide mapping method in 1986 (Fujiwara et al., 1986). Its precursor form was deduced from sequencing analysis of $\mathrm{H}$-protein cDNA isolated from chicken and human liver libraries (Yamamoto et al., 1991; Fujiwara et al., 1991; Koyata and Hiraga, 1991). The human H-protein precursor consists of 173 amino acids and the cofactor lipoic acid binds to ${ }^{107}$ Lys (Fujiwara et al., 1986).

Expression of the GCS. Expression of each component was scrutinized in chicken (Kure et al., 1991a). Overall activities of the GCS were detected in liver, kidney, and brain, but not in heart and spleen. P-protein mRNA was found only in liver, kidney, and brain. In contrast with that the transcripts of $\mathrm{H}$ - and 
T-proteins were detected in heart and spleen as well as liver, kidney and brain. Immunoblot analyses revealed that cross reactive material (CRM) against $\mathrm{H}$ - and $\mathrm{T}$-protein antibodies were present in heart and spleen. Furthermore, the component-specific activities for $\mathrm{H}$ - and $\mathrm{T}$-proteins were also detected in those two organs. Thus far, the organ specific localization of the GCS activities depends on the organ specific expression of P-protein gene. There was a strong positive correlation between the mRNA levels of $\mathrm{P}$ - and $\mathrm{H}$-proteins in liver, kidney, and brain, suggesting the coordinate expression mechanism of those two genes (Kure et al, 1991a).

\section{Biochemical basis of $N K H$}

Enzymatic analysis in liver. Defective GCS component of each case with NKH has been determined by measuring the individual activity of each component in our laboratory (Hayasaka et al., 1983, 1987; Tada, 1987). Until now we have analyzed 30 liver samples of biopsy and autopsy from NKH patients: 26 neonatal onset cases and 4 late onset cases. All liver samples from the neonatal cases had less than $1 \%$ of the overall GCS activities as compared with the control specimens, whereas $2-5 \%$ of the activities were detected in late onset cases. A total of 26 out of $30(87 \%)$ liver specimens had reduced activities of P-protein. The other 4 samples was found to be specifically deficit of T-protein activities. Two of the four late onset patients had the metabolic lesion in P-protein and the other two in T-protein.

Enzymatic analysis in lymphoblasts. As described in previous section the GCS is specifically expressed in liver, kidney, and brain and no enzymatic activities were found in easily-obtainable tissues such as fibroblasts or leukocytes. Liver biopsy is therefore, necessary for the enzymatic diagnosis of NKH. In addition, due to the difficulty in liver biopsy for healthy subjects, there has been no information about the GCS activities of healthy carriers for NKH. Recently, we developed a new assay method for the GCS (Kure et al., 1992a). The new method is based on the observation that low but detectable level of the GCS activity was present in the $\mathrm{EB}$ virus transformed lymphoblasts. In controls, the GCS activity ranged between 0.61 and $1.6 \mathrm{nmol}$ of ${ }^{14} \mathrm{CO}_{2}$ formed per hour per milligram protein. The average value was $0.94 \pm 0.27$, which was one fifth of that in control liver. In contrast, the GCS activity was undetectable in all 6 patients with P-protein deficiency. The results were in consistent with the GCS activities in their liver specimens of biopsy. The values of the GCS activity in lymphoblasts from the heterozygous parents averaged $0.35-0.11$, which was $38 \%$ of the normal controls. P-protein activity was also undetectable in lymphoblasts from the six patients. Thus, the lymphoblast assay should make it possible to diagnose the NKH patients enzymatically without risking the liver biopsy.

Immunochemical studies. Cross reactive material with anti-P-protein antibodies was analyzed by immunoblotting in three liver samples of autopsy (Kume 
et al., 1988). No detectable P-protein activities were detected in the two of three cases and the residual P-protein activity was found in the other case. In the two cases with neonatal onset no CRM was detected by immunoblotting. Reduced amount (3\% of the control livers) of CRM were observed in one patient with late onset NKH, which was equivalent with the residual activities of P-protein. No other immunochemical studies were so far reported.

\section{Molecular basis of $\mathrm{NKH}$}

Since P-protein gene is large and contains many exons, we at first analyzed its liver mRNA instead of its gene. A Japanese sib case with neonatal onset was investigated (Kure et al., 1991b). Total RNA was isolated from the liver specimens of autopsy and cDNA fragments were synthesized by reverse transcription mediated polymerase chain reaction (PCR), for sequencing analysis. Three base deletion was identified: the deletion resulted in the deletion of ${ }^{756} \mathrm{Phe}$. Expression analysis of the mutant P-protein cDNA in COS7 cells revealed that the three base deletion abolished the P-protein activities. The allele specific oligonucleotide hybridization experiment showed that the father did not carry the three-base deleted allele and the mother was heterozygous for the mutation. The liver cDNA fragment of the patients hybridized only with the mutant type oligo probe, suggesting that the patient's allele without the three-base deletion was not expressed in the patient's liver.

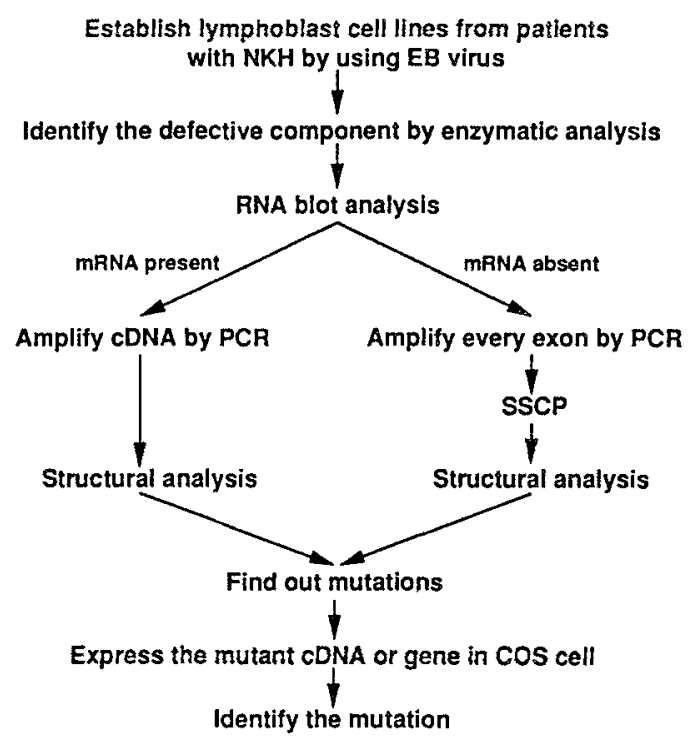

Fig. 1. Strategies for analyses of mutant P-protein gene. Our strategy for mutation detection was illustrated in flow chart. SSCP, single strand conformation polymorphism. 

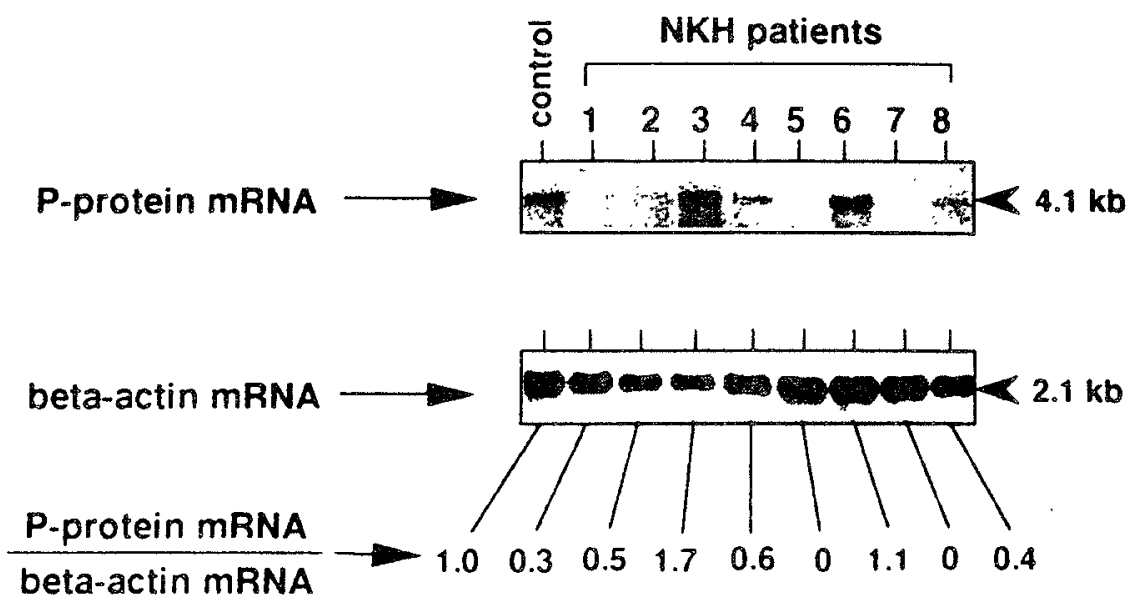

Fig. 2. Expression of P-protein mRNA in lymphoblasts from eight patients with P-protein deficiency. Polyadenylated RNA was isolated from eight lymphoblast cell lines established from the patients with P-protein deficiency. Five micrograms of polyadenylated RNA were size-separated by $2 \%$ formaldehyde agarose gel electrophoresis. The mRNAs were then transferred to nylon membrane and probed with ${ }^{32}$ P-labelled with human P-protein cDNA. The signals were visualized by autoradiography. To determine the relative amount of the P-protein mRNA $2.1 \mathrm{~kb} \beta$-actin mRNA signals were also detected as an internal control. Both P-protein and signal intensities of the $\beta$-actin mRNA were densitometrically measured and the relative ratios of $\mathrm{P}$-protein and $\beta$-actin mRNA levels were calculated.

Because of the infeasibility in access of liver specimens we made another strategy for mutant P-protein gene analysis (Fig. 1). The lymphoblasts established from the NKH patients provides the accessible source of mutant P-protein mRNA. We then analyzed P-protein mRNA levels in lymphoblasts from 8 patients with P-protein deficiency by Northern blot analysis (Fig. 2). As a result two cell lines expressed equivalent amount of P-protein mRNA with controls. Four patients had reduced levels of the mRNA and no mRNAs were detected in two cell lines. The results suggest that the genetic defects of P-protein gene may be heterogeneous among the neonatal cases.

According to the strategy described above we studied a NKH patient in Finland where the incidence of NKH is unusually high (Kure et al., 1992b). The activity of P-protein was not detected in the lymphoblasts of the patient, while P-protein mRNA of a normal size and level was present in the cells. Structural analysis of P-protein mRNA from the patient revealed a single nucleotide substitution from $G$ to $T$ in the protein coding region, which resulted in an amino acid alternation from Serine 564 to Isoleucine 564 (S564I mutation). No P-protein activity was detected when the mutant P-protein with this amino acid substitution was expressed in $\operatorname{COS} 7$ cells. The patient was homozygous for this mutation in his 
P-protein gene. Furthermore, this mutation was present in $70 \%$ (14 of 20) of P-protein gene alleles in Finnish patients with NKH. No allele with this mutation was found in 20 alleles of non-Finnish patients. The results suggest that this mutation is responsible for the high incidence of NKH in Finland. Two other mutations described above two missense mutations and one splicing error have been identified in Caucasian. All of them were found only in each affected family. Common mutations other than S564I have not yet been identified.

\section{Neurological basis of $\mathrm{NKH}$}

The neuropathogenesis of NKH remains largely uninvestigated. Glycine plays an important role not only in inhibitory neurotransmission in brainstem and spinal cord but also in excitatory neurotransmission in forebrain and cerebral cortex. To determine the localization of the GCS we cloned the brain cDNA clones encoding three components of rat GCS and perform in situ hybridization analysis in rat brain. The $\mathrm{H}$ - and T-protein mRNAs were widely distributed in olfactory bulbus, cerebrum, hippocampus, cerebellum, brainstem, and cord, while P-protein.mRNA was observed only in olfactory bulbus, cerebrum, hippocampus, and cerebellum (Kure et al., unpublished data). The distribution of P-protein mRNA was similar with that of a $N$-methyl-D-aspartic acid (NMDA) subtype glutamate receptor. The result was in line with those of the immunochemical analysis with anti-P-protein antibodies (Sato et al., 1991). The primary culture of rat cortex and hippocampus astrocytes expressed P-, H-, and T-protein mRNAs and had the GCS activities. Those observations suggest that the GCS in astrocytes may maintain the glycine concentration in the extracellular spaces around the NMDA receptor (Kure et al., 1995).

Because of those observation interest has focused on a new hypothesis of neuropathogenesis of NKH: The neurological disturbance associated with NKH is caused by glutamate neuroexcitotoxicity enhanced by high concentration of glycine through a NMDA receptor. As a clinical trial the NKH patients were given with the NMDA antagonists, ketamine (Ohya et al., 1991) and dextromethorphan (Hamosh et al., 1992). We treated three patients with dextromethorphan and found that dextromethorphan was effective in improvement of electroencephalogram (EEG) findings in two of three patients. But in one patient the treatment did not ameliorate the EEG nor the behavior findings. Figure 3 shows the EEG patterns of one patient who considerably responded the treatment. Despite the good response of the EEG to dextromethorphan therapy there was no apparent improvement in her developmental delay. We, therefore, think that the therapy is effective to some extent but far from satisfactory.

Recently, a new abnormality of amino acid metabolism was found in the brain of the patients with NKH. The D-serine concentration was markedly reduced in the cortical tissues of three $\mathrm{NKH}$ patients as compared with the control tissues (Iwama et al., 1997). D-serine is the only D-amino acid ever studied that 

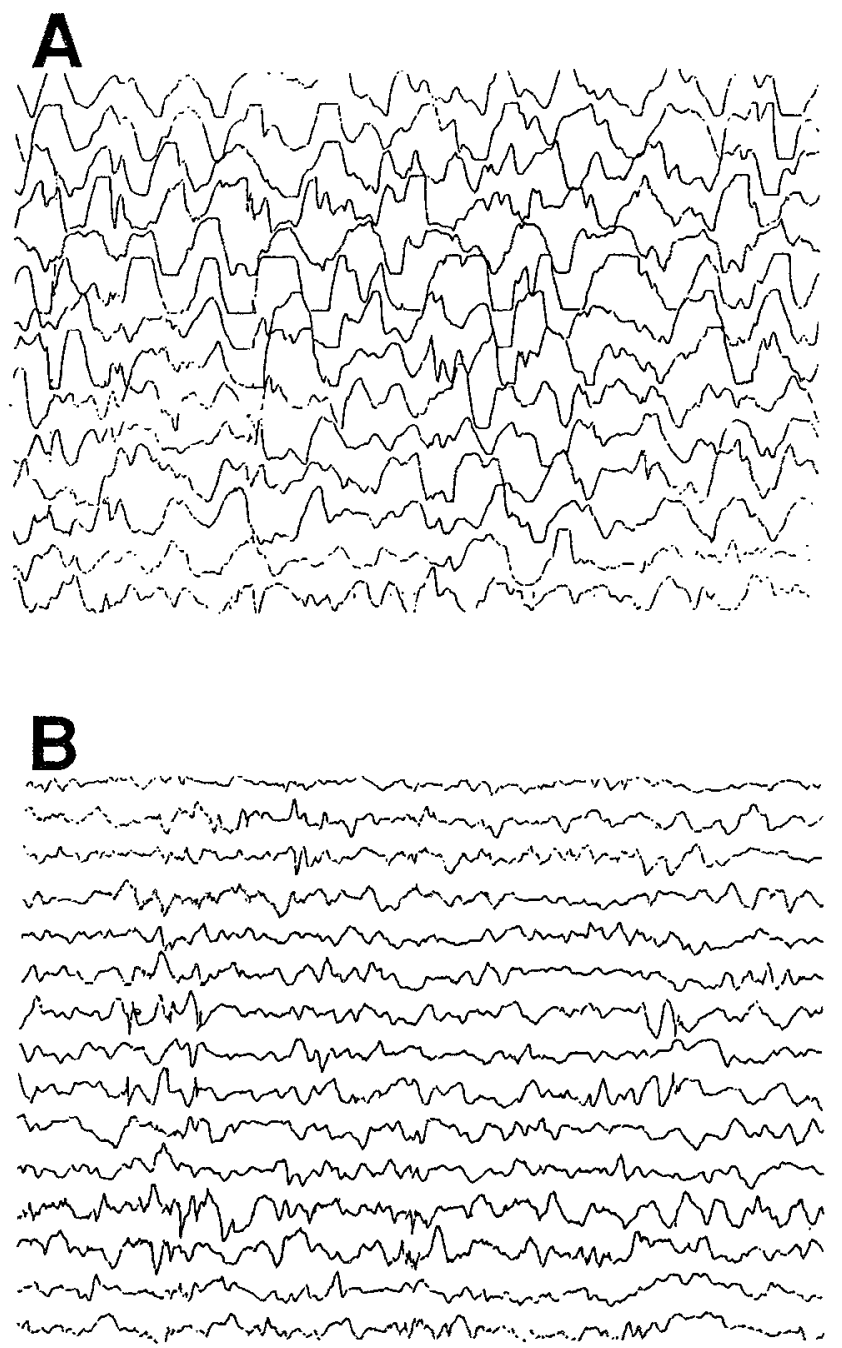

Fig. 3. Effect of NMDA antagonist on electroencephalogram of a patient with NKH. The patient had respiratory distress and hypotonia on 3 day after birth and received mechanical ventilation support. When she was 3 months old, she was given a diagnosis of NKH because of high glycine concentration in cerebrospinal fluid. No P-protein activity was detected in her lymphoblasts. An antagonist of NMDA receptor dextromethorphan was administered $(7.5 \mathrm{mg} /$ $\mathrm{kg}$ /day). After 3 days of the therapy it became possible for her to take milk by herself without a gastric tube. Effects of dextromethorphan on EEG finding are shown: panel A, before the treatment; panel B, 2 weeks after the initiation of therapy. Note the marked amelioration of EEG findings in panel $B$. 
naturally occurs in a free form at high concentration in the mammalian central nervus system. Because D-serine as well as glycine acts at the glycine modulatory site of NMDA receptor as potent agonist, D-serine may play a role in neuropathogenesis of NKH.

In conclusion we discussed here the pathogenesis of NKH from biochemical, molecular, and neurological view points. Further molecular and neurological studies are necessary for understanding of the neuropathogenesis and establishing the effective therapy of NKH.

\section{REFERENCES}

Craigen WJ (1996): Leigh disease with deficiency of lipoamide dehydrogenase: treatment failure with dichloroacetate. Pediatr Neurol 14: 69-71

Fujiwara K, Okamura-Ikeda K, Hayasaka K, Motokawa Y (1991): The primary structure of human $\mathrm{H}$-protein of the glycine cleavage system deduced by $\mathrm{cDNA}$ cloning. Biochem Biophys Res Commun 176: 711-716

Fujiwara K, Okamura-Ikeda K, Motokawa Y (1986): Chicken liver H-protein, a component of the glycine cleavage system. Amino acid sequence and identification of the $\mathrm{N}$ epsilonlipoyllysine residue. J Biol Chem 261: 8836-8841

Fujiwara K, Okamura-Ikeda K, Motokawa Y (1987): Amino acid sequence of the phosphopyridoxyl peptide from P-protein of the chicken liver glycine cleavage system. Biochem Biophys Res Commun 149: 621-627

Hamosh A, Johnston MV, Valle D (1995): Nonketotic hyperglycinemia. In: Scriver CR, Beaudet AL, Sly WS, Valle D (eds). The metabolic and molecular basis of inherited disease. McGraw-Hill, New York, pp 1337-1348

Hamosh A, McDonald JW, Valle D, Francomano CA, Niedermeyer E, Johnston MV (1992): Dextromethorphan and high-dose benzoate therapy for nonketotic hyperglycinemia in an infant. J Pediatr 121: $131-135$

Hayasaka K, Nanao K, Takada G, Okamura-Ikeda K, Motokawa Y (1993): Isolation and sequence determination of cDNA encoding human T-protein of the glycine cleavage system. Biochem Biophys Res Commun 192: 766-771

Hayasaka K, Tada K, Fueki N, Nakamura Y, Nyhan WL, Schmidt K, Packman S, Seashore MR, Haan E, Danks DM, et al. (1987): Nonketotic hyperglycinemia: analyses of glycine cleavage system in typical and atypical cases. J Pediatr 110: 873-877

Hayasaka K, Tada K, Kikuchi G, Winter S, Nyhan WL (1983): Nonketotic hyperglycinemia: two patients with primary defects of P-protein and T-protein, respectively, in the glycine cleavage system. Pediatr Res 17: 967-970

Iwama H, Takahashi K, Kure S, Hayashi F, Narisawa K, Tada K, Mizoguchi M, Takashima S, Tomita U, Nishikawa T (1997): Depletion of cerebral D-serine in non-ketotic hyperglycinemia: possible involvement of glycine cleavage system in control of endogenous D-serine. Biochem Biophys Res Commun (in press)

Kikuchi G (1973): The glycine cleavage system: composition, reaction mechanism, and physiological significance. Mol Cell Biochem 1: $169-187$

Kikuchi G, Hiraga K, Yoshida T (1980): Role of the glycine-cleavage system in glycine and serine metabolism in various organs. Biochem Soc Trans 8: 504-506

Koyata H, Hiraga K (1991): The glycine cleavage system: structure of a cDNA encoding human H-protein, and partial characterization of its gene in patients with hyperglycinemias. Am $\mathrm{J}$ Hum Genet 48: $35 \uparrow-361$

Kume A, Koyata H, Sakakibara T, Ishiguro Y, Kure S, Hiraga K (1991): The glycine cleavage system. Molecular cloning of the chicken and human glycine decarboxylase cDNAs and

Vol. 42, No. 1, 1997 
some characteristics involved in the deduced protein structures. J Biol Chem 266: 3323-3329

Kume A, Kure S, Tada K, Hiraga K (1988): The impaired expression of glycine decarboxylase in patients with hyperglycinemias. Biochem Biophys Res Commun 154: 292-297

Kure S, Koyata H, Kume A, Ishiguro Y, Hiraga K (1991a): The glycine cleavage system. The coupled expression of the glycine decarboxylase gene and the $\mathrm{H}$-protein gene in the chicken. J Biol Chem 266: 3330-3334

Kure S, Narisawa K, Tada K (1991b): Structural and expression analyses of normal and mutant mRNA encoding glycine decarboxylase: Three-base deletion in mRNA causes nonketotic hyperglycinemia. Biochem Biophys Res Commun 174: 1176-1182

Kure S, Narisawa K, Tada K (1992a): Enzymatic diagnosis of nonketotic hyperglycinemia with lymphoblasts. J Pediatr 120: 95-98

Kure S, Narisawa K, Tada K (1995): The glycine cleavage system in astrocytes: Its physiological role revealed by study of hyperglycinemia. I Neurochem 65 : S125

Kure S, Takayanagi M, Narisawa K, Tada K, Leisti I (1992b): Identification of a common mutation in Finnish patients with nonketotic hyperglycinemia. J Clin Invest 90: 160-164

Motokawa Y, Kikuchi G (1972): Isolation and partial characterization of the components of the reversible glycine cleavage system of rat liver mitochondria. J Biochem 72: 1281-1284

Nanao K, Takada G, Takahashi E, Seki N, Komatsu Y, Okamura-Ikeda K, Motokawa Y, Hayasaka K (1994): Structure and chromosomal localization of the aminomethyltransferase gene (AMT). Genomics 19: 27-30

Ohya Y, Ochi N, Mizutani N, Hayakawa C, Watanabe K (1991): Nonketotic hyperglycinemia: Treatment with NMDA antagonist and consideration of neuropathogenesis. Pediatr Neurol 7: $65-68$

Okamura-Ikeda K, Fujiwara K, Yamamoto M, Hiraga K, Motokawa Y (1991): Isolation and sequence determination of cDNA encoding $\mathrm{T}$-protein of the glycine cleavage system. $\mathrm{J}$ Biol Chem 266: 4917-4921

Okamura-Ikeda K, Ohmura Y, Fujiwara K, Motokawa Y (1993): Cloning and nucleotide sequence of the gcv operon encoding the Escherichia coli glycine-cleavage system. Eur $\mathrm{J}$ Biochem 216: 539-548

Sato K, Yoshida S, Fujiwara K, Tada K, Tohyama M (1991): Glycine cleavage system in astrocytes. Brain Res 567: 64-70

Tada K (1987): Nonketotic hyperglycinemia: clinical and metabolic aspects. Enzyme 38: 27-35

Tada K, Hayasaka K (1987): Nonketotic hyperglycinemia: clinical and biochemical aspects. Eur J Pediatr 146: 221-227

Tada K, Narisawa K, Yoshida T, Yokoyama K, Nakagawa H, Tanno K, Mochizuki K, Arakawa T, Yoshida T, Kikuchi $G$ (1969): Hyperglycinemia: a defect in glycine cleavage reaction. Tohoku J Exp Med 98: 289-296

v. Wendt L, Hirvasniemi A, Similä S (1979): Nonketotic hyperglycinemia: A genetic study of 13 Finish families. Clin Genet 15: 411-417

Yamamoto M, Koyata H, Matsui C, Hiraga K (1991): The glycine cleavage system: Occurrence of two types of chicken $H$-protein mRNAs presumably formed by the alternative use of the polyadenylation consensus sequences in a single exon. J Biol Chem 266: 3317-3322 